\title{
Why Variability Facilitates Spinal Learning
}

\author{
Matthias D. Ziegler, ${ }^{1}$ Hui Zhong, ${ }^{1}$ Roland R. Roy, ${ }^{1,3}$ and V. Reggie Edgerton ${ }^{1,2,3}$ \\ Departments of ${ }^{1}$ Integrative Biology and Physiology and ${ }^{2}$ Neurobiology and ${ }^{3}$ Brain Research Institute, University of California, Los Angeles, Los Angeles, \\ California 90095
}

\begin{abstract}
Spinal Wistar Hannover rats trained to step bipedally on a treadmill with manual assistance of the hindlimbs have been shown to improve their stepping ability. Given the improvement in motor performance with practice and the ability of the spinal cord circuitry to learn to step more effectively when the mode of training allows variability, we examined why this intrinsic variability is an important factor. Intramuscular EMG electrodes were implanted to monitor and compare the patterns of activation of flexor (tibialis anterior) and extensor (soleus) muscles associated with a fixed-trajectory and assist-as-needed (AAN) step training paradigms in rats after a complete midthoracic (T8-T9) spinal cord transection. Both methods involved a robotic arm attached to each ankle of the rat to provide guidance during stepping. The fixed trajectory allowed little variance between steps, and the AAN provided guidance only when the ankle deviated a specified distance from the programmed trajectory. We hypothesized that an AAN paradigm would impose fewer disruptions of the control strategies intrinsic to the spinal locomotor circuitry compared with a fixed trajectory. Intrathecal injections of quipazine were given to each rat to facilitate stepping. Analysis confirmed that there were more corrections within a fixed-trajectory step cycle and consequently there was less coactivation of agonist and antagonist muscles during the AAN paradigm. These data suggest that some critical level of variation in the specific circuitry activated and the resulting kinematics reflect a fundamental feature of the neural control mechanisms even in a highly repetitive motor task.
\end{abstract}

\section{Introduction}

Recovery of hindlimb motor function after a complete spinal cord transection at a midthoracic level in adult animals can be improved with bipedal step training on a moving treadmill system (for review, see Edgerton et al., 2006). Using a variety of algorithms, it was shown that robotic assistance during step training in combination with the administration of the $5-\mathrm{HT}_{2}$ agonist quipazine enhanced this training effect in mice that were spinal cord transected as adults (Fong et al., 2005). The consistency of effective stepping also can be improved by manually assisting the limb movement as is needed to sustain stepping during the training sessions in neonatal rats (Kubasak et al., 2008). Although this approach has been successful, there has been no quantitative analysis of this strategy or what is meant by manual assistance. We have demonstrated previously that some training effects can be achieved in complete spinal mice when robotic arms are used to impose a fixed trajectory at the ankle, followed by brief periods of no assistance (Fong et al., 2005). The fixed trajectory, however, ignores the importance of variation in motor unit activation patterns, and thus step kinematics, as a feature of neural control (Hausdorff, 2005). This could result in constantly correcting the intrinsically defined stepping patterns

\footnotetext{
Received April 16, 2010; revised May 25, 2010; accepted June 16, 2010.

This work was supported by the National Institute of Neurological Disorders and Stroke Grant NS054159 and the California Roman Reed Bill. We thank Maynor Herrera for providing excellent animal care and surgical support, Sharon Zdunowski for her technical support, Lance Cai and Andy Fong for their help in developing the robotic system, and Niranjala Tillakaratne for her constructive guidance and valuable discussions.

Correspondence should be addressed to Dr. V. Reggie Edgerton, Department of Integrative Biology and Physiology, University of California, Los Angeles, 621 Charles E. Young Drive, Los Angeles, CA 90095-1527. E-mail: vre@ucla.edu.

DOI:10.1523/JNEUROSCI.1938-10.2010

Copyright $\odot 2010$ the authors $\quad 0270-6474 / 10 / 3010720-07 \$ 15.00 / 0$
}

that would not likely be programmed by the robot. Conversely, minimizing the variation in the naturally occurring muscle activation patterns may result in habituation to the sensory input and a reduced response to step training (Hidler and Wall, 2005; Cai et al., 2006).

Using robotic training, spinal mice learned to step more effectively when continuous, but soft, assistance was provided compared with a fixed trajectory imposed on the ankle (Cai et al., 2006). We hypothesized that the abolishment of variation within and between steps (fixed-trajectory paradigm) would result in a greater disruption of the neural control strategies that are inherent in the spinal locomotor circuitry and would cause a suboptimal training paradigm when compared with an assist-as-needed (AAN) paradigm that would allow step-to-step variability (Jezernik et al., 2003; Hidler and Wall, 2005; Wirz et al., 2005; Cai et al., 2006; Christou et al., 2007; Emken et al., 2008).

In the present study, we compared the patterns of activation of flexor and extensor motor pools during an AAN algorithm with the imposition of a fixed-trajectory algorithm in complete spinal transected adult rats. Soleus and tibialis anterior (TA) muscle activity during bipedal stepping of intact rats show alternating bursts with little or no coactivation between the muscles (Courtine et al., 2009). We observed a significant disruption of the normal cyclic pattern of excitation of flexor and extensor motor pools during the fixed-trajectory control paradigm, whereas the AAN paradigm resulted in a more normal flexorextensor activation pattern. These results have important implications regarding the fundamental features of the neural control of locomotion as well as to the robotic control strategies that will be most effective for the recovery of locomotor ability after a spinal cord injury. 


\section{Materials and Methods}

Animals. Data were collected from 12 adult Wistar Hannover female rats ( $\sim 7$ months of age). All procedures were approved by the Chancellor's Animal Research Committee at the University of California, Los Angeles and followed the American Physiological Society Animal Care Guidelines.

Experiment timeline. Before the spinal cord transection surgery, the rats were placed on the treadmill belt with the robot (passive mode) attached to the ankles, and step trajectories were recorded. The transection surgery was performed when the rats were between 2 and 3 months old. Although the rats were not step trained, they were exposed to the treadmill once a month after transection for acclimation to the testing setup and to determine whether they had recovered any stepping ability without training. None of the rats could step spontaneously. Chronic intramuscular EMG electrodes were implanted at 3-4 months after transection, i.e., when the rats were 6-7 months old. The rats were tested 1 month after the EMG surgery, i.e., 5 months after the transection surgery, when they were 7-8 months old. The rats were tested using the fixedtrajectory and AAN paradigms for a single 20 min period during which data were recorded.

Spinal cord transection. All surgical procedures were performed under aseptic conditions and with the rats deeply anesthetized using isoflurane gas via facemask as needed. The spinal cords of 10- to 12-week-old rats were transected completely at spinal cord level T8-T9 as described previously (Kubasak et al., 2008). The paravertebral muscles were retracted, a partial laminectomy of the T8 and T9 vertebrae was performed, and the dorsal dura was opened with an " $\mathrm{H}$ " cut to expose the spinal cord. The spinal cord then was completely transected with microscissors. Two surgeons independently verified a complete transection by passing a glass probe through the site and lifting both cut ends of the spinal cord. The paravertebral muscles and fascia were sutured using 4-0 chromic gut, and the skin incisions were closed with 4-0 Ethilon suture.

After surgery, the rats were placed in an incubator maintained at $37^{\circ} \mathrm{C}$ until fully recovered. The rats were given lactated Ringer's solution $(5 \mathrm{cc}$, s.c.) to prevent dehydration. Baytril $(0.2 \mathrm{cc}$, i.m., twice daily; enrofloxacin; Bayer HealthCare), a general antibiotic, and buprenex $(0.05 \mathrm{mg} / \mathrm{kg}$, s.c., twice daily; buprenorphine hydrochloride; Reckitt Benckiser Healthcare), an analgesic, were administered during the first $2 \mathrm{~d}$ after surgery. Manual bladder expressions were performed three times per day for the first week and then twice daily, at $12 \mathrm{~h}$ intervals, thereafter. Rats were housed individually in polycarbonate cages in a room maintained at $26 \pm 1^{\circ} \mathrm{C}$ with $40 \%$ humidity and a $12 \mathrm{~h}$ light/dark cycle. Dry kibble and water were provided ad libitum, and pieces of fruit were given daily.

Intramuscular EMG implants. Three months after transection, intramuscular EMG electrodes were implanted in the soleus (an ankle extensor) and TA (an ankle flexor) muscles bilaterally. A skin incision was made along the sagittal suture of the skull, and the connective tissue covering the skull were separated. The skull was thoroughly dried, and four stainless steel screws were firmly inserted into the exposed bone. An amphenol connector was placed between the screws and rigidly affixed to the bone using dental cement. Multistranded, Teflon-coated stainless steel wires (AS632; Cooner Electronics) connected to gold-plated amphenol pins in the recording unit were passed subcutaneously to the lower leg. Pairs of wires were passed into each muscle belly using a 23 gauge needle, and a small notch $(\sim 0.5-1.0 \mathrm{~mm})$ was made in the Teflon coating to make the electrodes. Each muscle was stimulated through the head connector to ensure proper placement of the electrodes. The electrode wires then were anchored at both ends with 4-0 Ethilon suture. All exposed areas were kept moist with $0.9 \%$ saline washes. After implantation, all exposed muscle areas was lavaged with a saline-betadine solution, and the muscle, fascia, and skin were sutured in layers.

Intrathecal catheter implants. During the same surgery, the rats were implanted with intrathecal catheters. A length of polyurethane tubing (PE-10) was passed through a plastic head connector that was placed between two screws inserted into the exposed skull. A $4 \mathrm{~cm}$ length of tubing was left at the proximal end to be used for the injections. The distal end of the tubing had a tip made of flexible SILASTIC tubing to avoid damaging the spinal cord. A partial laminectomy was performed at vertebral level T11, and the SILASTIC tip was carefully placed in the subarachnoid space and then tunneled to spinal level L2-L3. The catheter was secured with suture at its entrance into the vertebral column. The exposed tubing at the proximal end was sealed with a metal plug to prevent contamination. The tubing was flushed with sterile saline three times per week with a volume of saline equal to the length of the tubing. Before each flushing or each injection of quipazine (see below), the tubing was wiped with $70 \%$ alcohol.

Quipazine administration. Quipazine was administered at a dosage of $0.05 \mathrm{mg} / \mathrm{kg}$ at $\sim 5 \mathrm{~min}$ before testing (De Leon and Acosta, 2006). A volume of $\sim 20 \mu \mathrm{l}$ of quipazine solution (diluted in sterile water at 1 $\mathrm{mg} / \mathrm{ml}$ ) was injected into the intrathecal tubing, followed by injection of $20 \mu \mathrm{l}$ of sterile saline.

Computer-controlled robotic trajectory paradigms. After recovery from the transection surgery, the rats were placed in a body harness to stand bipedally on a treadmill, and both ankles of the rats were attached to robotic arms by a rubber ankle loop secured by an alligator clip. Because of orthopedic-related changes that occur in spinal rats after prolonged periods, the most effective stepping kinematics are usually not synonymous with the best stepping kinematics of the same rat before the complete spinal cord transection. Therefore, to identify the most appropriate trajectory to use for training, an experienced trainer manually moved the limbs of each rat through a typical step trajectory that was recorded by the robotic arms while in a passive mode. The most common trajectory pattern observed across rats from these training sessions was selected to train all rats. Five months after the spinal cord transection, the rats were tested using one or two control strategies: a fixed-trajectory paradigm (fixed recording supplemental Movie and supplemental Movie legends, available at www.jneurosci.org as supplemental material) that allowed little variance between steps and an AAN paradigm (fixed recording supplemental Movie and supplemental Movie legends, available at www.jneurosci.org as supplemental material) that provided robotic guidance when the ankle deviated a specified distance from the programmed trajectory, thus allowing for some variance in the programmed trajectory. Eight rats were tested with the fixed-trajectory paradigm. These same rats were tested 2 weeks later with the AAN paradigm (AAN1). To control for the possibility that the initial testing with the fixed trajectory may have affected the response to the AAN paradigm, a separate group of eight rats were tested only with the AAN paradigm (AAN2).

During the fixed-trajectory testing, the robotic arms were constantly in an active mode, consistently repeating the ankle trajectory recorded by the animal trainer. This algorithm causes the computer to move the robotic arm to the desired position in the trajectory, and, once it has reached that position, it moves to the next trajectory point (Fig. $1 A$ ). This process is repeated until a full trajectory is completed at which time it starts over from the initial position. During this testing paradigm, the robot is continuously correcting the position of the ankle.

In contrast, the AAN algorithm creates a desired window around the trajectory that moves at a predetermined speed (Fig. $1 B$ ). When the ankle position is within the window, there is a small constant velocity field tangent to the desired trajectory that biases the motion of the robot but without spatial or temporal enforcement (Cai et al., 2006). When the ankle position falls outside the window, there is a radial velocity field that guides the robotic arm into the window. This force is proportional to the distance from the center of the window. Therefore, when the ankle is outside a given distance from the desired position, the robotic arm guides it back, but if the ankle is within the window, there is only a slight directional force to provide timing of the step. The final trajectories that occur during the AAN training can vary greatly between animals. Although there are significant differences during the swing phase, the activity during the stance phase is similar between the paradigms as the programmed trajectory matches the position of the ankle for the specific treadmill speed.

Flexor-extensor coordination analyses. Analysis of the EMG bursting between legs was done in individual animals by plotting joint probability 
distributions (JPDs) (see Fig. 5A) and between groups by determining the coordination coefficient (see Fig. 5B) for each animal. Each step in a $20 \mathrm{~s}$ period was normalized to a specific length and averaged. Each point of the JPD represents a single point in the step cycle of this averaged step. The coordination coefficient was determined by measuring the distance of each point in the JPD from the nearest axis: given each point $\{a, b\}$ the distance to the nearest axis is minimum $(a, b)$. Points were used only when at least one muscle was active and were discarded when both muscles were below a threshold for activity, determined to be anything below twice the SD of the background noise.

Statistical analyses. Kinematics and EMG data are reported as mean \pm SEM. One-way ANOVA was used to determine overall differences, and Tukey's post hoc tests were used to compare the three training groups within each training day. $p<0.05$ was used to define statistical significance.

\section{Results}

\section{Step trajectories}

Comparisons of the step trajectory and EMG responses for the same animal during a $30 \mathrm{~s}$ fixed-trajectory versus AAN1 session are shown in Figure 2. There is a narrower band of ankle positions during the swing phase with the fixed-trajectory (Fig. $2 A$ ) compared with the AAN1 (Fig. $2 B$ ) paradigm. The average variance in position for each rat was measured at midswing (Fig. $3 A$ ). The position variance in the fixed trajectory $(3.20 \pm 0.37 \mathrm{~mm})$ was less than half the size of the position variance in the AAN1 $(7.44 \pm 0.74 \mathrm{~mm})$ and AAN2 $(6.15 \pm 0.58 \mathrm{~mm})$ groups. In the example shown in Figure 2, the ankle moves through a larger trajectory with the AAN1 (Fig. $2 B$ ) than the fixed-trajectory (Fig. 2A) paradigm.

During the swing phase, there were more robotic corrections to the step trajectory with the fixed compared with the AAN1 algorithm (examples of changes are shown by arrows in Fig. $2 \mathrm{~A}$ vs $B)$. The average number of robotic adjustments during the swing phase was approximately threefold greater in the fixed-trajectory (12.7 \pm 1.6$)$ compared with the AAN1 $(4.4 \pm 0.4)$ and AAN2 (4.75 \pm 0.86$)$ groups (Fig. 3B).

The size of the step cycle during AAN was dependent on the individual rat. The mean vertical displacement during the fixedtrajectory $(17.1 \pm 0.48 \mathrm{~mm}), \mathrm{AAN} 1(18.8 \pm 2.8 \mathrm{~mm})$, and AAN2 $(19.8 \pm 3.2 \mathrm{~mm})$ groups was similar (Fig. 3C). The horizontal displacement was smaller in the fixed-trajectory $(35.89 \pm 0.67$ $\mathrm{mm})$ than the AAN1 $(40.62 \pm 1.17 \mathrm{~mm})$ and AAN2 $(41.6 \pm 1.2$ $\mathrm{mm}$ ) groups (Fig. 3D). Generally, these differences reflect the higher variation in the step trajectory allowed with the AAN compared with the fixed-trajectory algorithm.

\section{EMG activation patterns}

The soleus and TA muscles showed distinct, alternating EMG bursts with the AAN algorithm, whereas there was a high amount of coactivation of these two antagonists with the fixed-trajectory algorithm (Fig. 2C). The soleus was active for a higher percentage of the step cycle with the fixed $(72.72 \pm 5.9 \%)$ than the AAN1 $(12.53 \pm 3.87 \%)$ and AAN2 (22.33 $\pm 6.97 \%)$ groups (Fig. 4 A). A lower percentage of the soleus activity occurred during the swing phase with the AAN1 (24.54 $\pm 5.0 \%)$ and AAN2 (33.6 \pm $6.3 \%)$ groups than the fixed-trajectory paradigm (51.19 \pm
6.6\%) (Fig. 4C). The TA also was active for a higher percentage of the step cycle with the fixed-trajectory $(25.04 \pm 4.0 \%)$ than the AAN1 (12.7 $\pm 1.7 \%)$ and AAN2 (11.7 $\pm 3.8 \%)$ paradigms (Fig. $4 B$ ). The percentage activation of the TA during the swing phase, however, was similar for all groups (Fig. 4D).

\section{Total muscle activation}

Although both the TA and soleus muscles were activated for a longer period of time during each step cycle with the fixed than the AAN trajectory algorithm (Fig. $4 A, B$ ), the mean integrated EMG was similar for the two muscles within each group (Fig. $4 E, F)$. Thus, the AAN algorithm results in distinct alternating high-amplitude bursts, whereas the fixed-trajectory algorithm results in prolonged highly coactivated low-amplitude bursts throughout the stepping sessions.

\section{Flexor-extensor coactivation}

There was a higher amount of coactivation between the antagonistic muscles during the fixed-trajectory than the AAN paradigm (Fig. 2C,D). This is clearly illustrated by the JPDs in Figure 5: there is clear reciprocal activation of the soleus and TA with the AAN (Fig. $5 B$ ) but not the fixed-trajectory (Fig. $5 A$ ) paradigm. In addition, the coordination coefficient was higher in the fixedtrajectory $(0.21 \pm 0.10)$ compared with the AAN1 $(0.08 \pm 0.01)$ and the AAN2 $(0.14 \pm 0.05)$ groups.

\section{Discussion}

The present results demonstrate that, when the intrinsic variability of the spinal locomotor circuitry is accommodated, the patterns of coordination between hindlimb flexor and extensor muscles are more normal than when a fixed trajectory is imposed. Based on studies of locomotor circuits, step-to-step variations reflect a stochastic phenomenon within both sensory and motor circuits (Rossignol, 2000; Kiehn, 2006; Dominici et al., 2007; Kozlov et al., 2009). This variation in stepping implies that the neural control system is based on processes within a network of neurons that interpret the sensory information during stepping and based on this input make decisions on the appropriate motor responses. The importance of the sensory system 


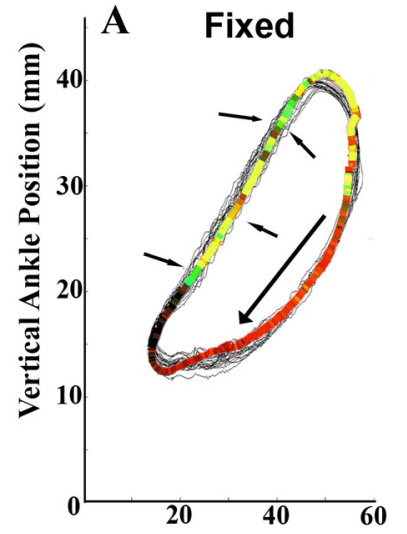

Horizontal Ankle Position (mm)

C
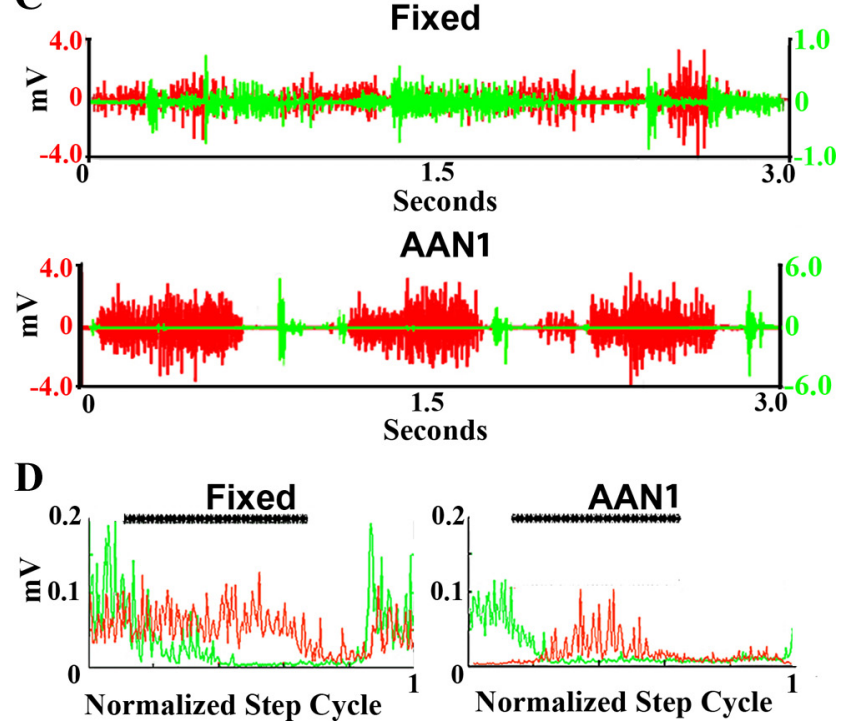

Figure 2. $\quad \boldsymbol{A}$ (Fixed) and $\boldsymbol{B}$ (AAN1) show the ankle trajectories recorded over $30 \mathrm{~s}$ of movement for the fixed-trajectory and the AAN1 paradigms, respectively. The colored areas represent the average EMG activity recorded from the soleus and TA muscles: red, soleus activity; green, TA activity; yellow, soleus and TA muscle coactivation. The intensity of the color is directly proportional to the amplitude of the EMG signal. The arrows point to examples of changes in direction attributable to the robotic arm guiding the ankle toward the trajectory. C shows the raw EMG activity of the soleus and TA muscles during $3.0 \mathrm{~s}$ of movement for each paradigm (top, Fixed; bottom, AAN1). D shows the averaged integrated EMG for the soleus (red) and TA (green) muscles from over 30 continuous seconds of stepping for each paradigm (left, Fixed; right, AAN1). The ${ }^{* * * *}$ section represents the stance phase of the step cycle.

has been seen in rats with a complete spinal cord transection in which the stepping rate is modulated to directly match the speed of the treadmill and the activation of motor pools are modulated to a change in the load placed on the limbs during stepping (Edgerton et al., 1991; De Leon et al., 1998; Timoszyk et al., 2005; Frigon and Rossignol, 2006; Giszter et al., 2008; Ichiyama et al., 2008). We predicted that a lack of variation in the step cycles during the fixed-trajectory paradigm would impose continuous interruptions causing interference with the normal progression of activation that the networks planned to execute, and this would result in an inability to learn or improve the performance of the motor task.

Conversely, the sensitivity to sensory input may decrease as a result of the lack of variation with a fixed-trajectory paradigm. In turn, this desensitization to the sensory input would
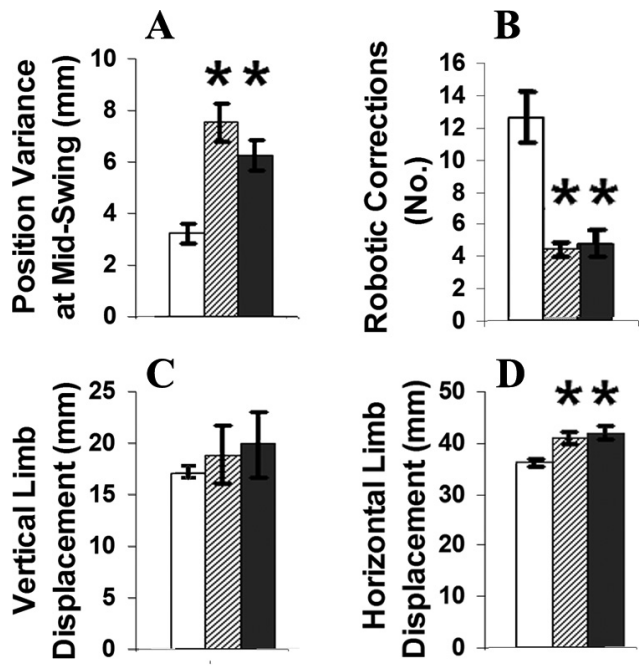

$\square$ Fixed $\square$ AAN1 $\square$ AAN2

Figure 3. A shows the average position variance at midswing. $\boldsymbol{B}$ shows the mean number of robotic corrections during the swing phase for the fixed-trajectory and AAN1 groups, as well as a second group of animals tested only under AAN conditions (AAN2). $\boldsymbol{C}$ and $\boldsymbol{D}$ are the average vertical and horizontal displacement during the different training paradigms. Values are mean \pm SEM for 20 s of movement for eight rats per group. ${ }^{*} p<0.05$, significantly different from fixed-trajectory group.
Soleus

EMG Activity

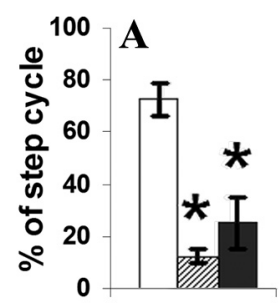

C
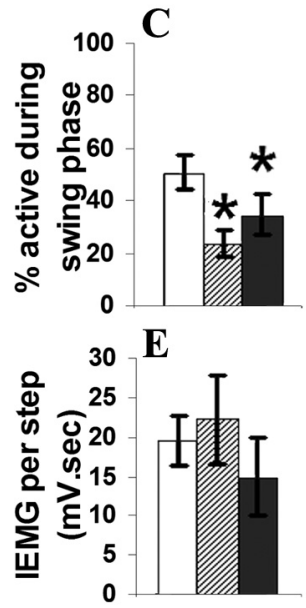

TA

EMG Activity

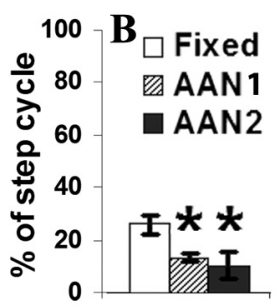

D
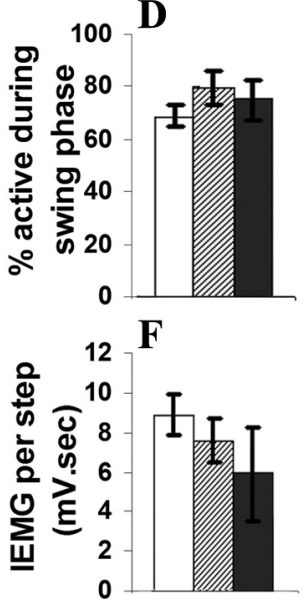

Figure 4. $\quad \boldsymbol{A}$ and $\boldsymbol{B}$ show the percentage of the entire step cycle that the EMG bursting is present in the soleus and TA muscles within the fixed-trajectory and AAN paradigm groups. $C$ and $\boldsymbol{D}$ show the duration of the EMG bursting that occurs during the swing phase of the step. $\boldsymbol{E}$ and $\boldsymbol{F}$ show the integral of the EMG during the entirestep cycle. Values are mean \pm SEM for 20 s of movement for eight rats per group. ${ }^{*} p<0.05$, significantly different from the fixed-trajectory group.

result in a decrease in the activity within the sensorimotor and spinal neural control systems (Wirz et al., 2005). In fact, a lack of variation in stepping during a fixed robotic trajectory training paradigm results in a decrease in the EMG activity in the 
biceps femoris and medial gastrocnemius after only a few minutes of training in chronically injured patients (Dietz and Müller, 2004). In the current study, we do not see this decrease in EMG activity during one training session with the robot during either paradigm.

\section{EMG/trajectory coordination}

In the present study, the fixed-trajectory paradigm continuously forced the legs toward a trajectory that would differ from the one that would have been selected by a stochastic system. The continual forced adjustments to the leg into a predetermined pattern resulted in the soleus and TA motor pools being in a continuous state of correcting throughout the swing phase. Similar to other studies that reported increases in the activation of the medial gastrocnemius and hamstring muscles during the swing phase of a fixed-trajectory robotic training session (Hidler and Wall, 2005; Israel et al., 2006), we observed an increase in soleus activity during the swing phase of the fixed-trajectory compared with the AAN-trajectory paradigm. The increased out-of-phase activation of the soleus and the increased TA activity during the swing phase imply that it occurred as a result of "unexpected" sensory input caused by the robot forcing adjustments away from the "planned" step trajectory. This interpretation itself implies a feedforward mechanism within the spinal locomotor circuitry (Edgerton et al., 2008).

\section{Muscle coactivation}

The soleus and TA muscles are antagonists, i.e., an ankle extensor and flexor, respectively. During bipedal stepping of intact rats, there is little to no coactivation between these antagonists. The fixedtrajectory algorithm results in continuous adjustments during the swing phase that trigger simultaneous bursting in the two muscles (Fig. 2). When the rat is allowed a critical level of variation of the trajectory within and between steps with the AAN paradigm, this simultaneous activity (coactivation) between muscles occurs rarely.

\section{Neural control}

The process of learning to step with a significantly modified neural circuitry, e.g., loss of all supraspinal input after a complete spinal cord transection, seems analogous to a naive circuit learning a new motor skill. Learning a motor skill is usually associated with a reduction in the variability of performing the task. Studies on neuronal signaling have shown that intrinsic activities, such as "noise" from synaptic processes, as well as extrinsic sources, including sensory input, contribute to the variability in neuronal activity (Stein et al., 2005; Faisal et al., 2008). Although in some cases variability in a signal is thought of as detrimental noise that may disrupt information that is being transmitted and needs to be eliminated (Bialek et al., 1991; Shadlen and Newsome, 1998), other studies have shown evidence of the importance of neuronal variability when learning new motor output techniques. Neurons in the motor cortices of monkeys learning to perform seemingly repetitive tasks have high variability in spiking activity (Mandelblat-Cerf et al., 2009). Similarly, variability is seen in neurons in the forebrain nucleus of songbirds that are learning a new song (Kao et al., 2008). In both of these cases, the variability decreases as the animal learns the task (Kao et al., 2008;

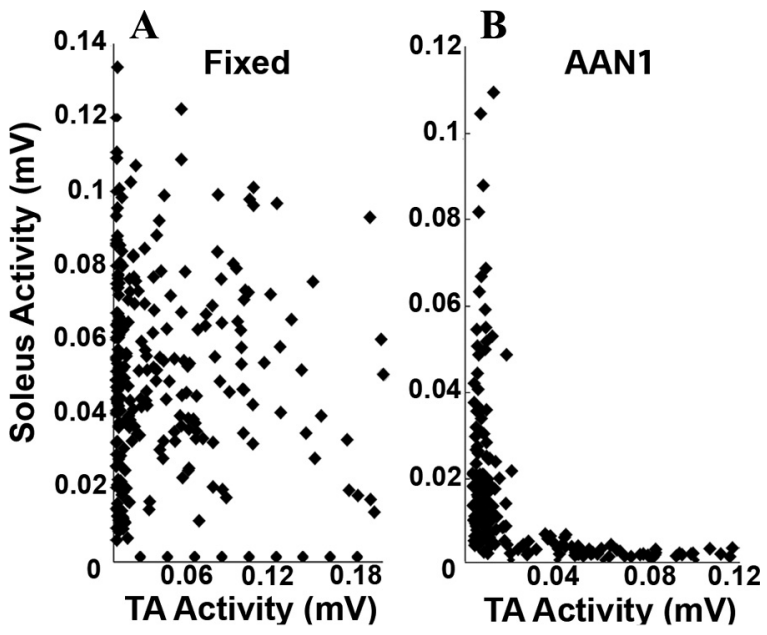

Figure 5. $\quad \boldsymbol{A}$ and $\boldsymbol{B}$ show examples of joint probability distributions (see Materials and Methods) comparing the EMG of the soleus and TA muscles for the fixed-trajectory and AAN paradigms, respectively, of a single rat. The coordination coefficient for this rat was 0.15 during the fixed trajectory and 0.04 during the AAN testing. Values in all graphs are from $20 \mathrm{~s}$ of movement for eight rats per group.

Figure 6. A theoretically ideal robotic AAN system will evaluate the robotic/animal interactions after each step. The AAN system Small Window with Ideal Window Size with Large Robotic Intervention Moderate Robotic Intervention

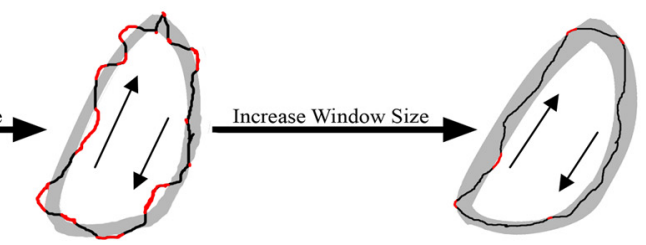
will start with a large window in which the robot is passive (left). After a step is completed (black line), the system will check how many times and during what part of the step cycle the robotic system was activated (red). If the robot is activated below some reevaluate. The middle example shows a high level of robotic activity (red), and the response is to increase the size of the window (right). This system will continue to change after each step cycle.

Mandelblat-Cerf et al., 2009). Improved consistency in EMG activity during AAN testing in addition to the previously reported improved stepping ability after training with an AAN paradigm (Cai et al., 2006) are consistent with this variability being a fundamental feature of the learning that occurs in the spinal circuitry that controls locomotion after a spinal cord injury (Edgerton et al., 2008).

The phenomenon of nonlinear noise that results in improved motor output signaling in an AAN mode has the features of stochastic resonance (McDonnell and Abbott, 2009). Stochastic resonance is when noise has the ability to enhance the signal processing as opposed to degrading the signal. Signal noise may improve the learning by allowing the enhancement of those synaptic connections that occur in a spike-timing-dependent plasticity (STDP) manner. This synaptic modification is based on the concept that neuronal synapses are either strengthened or weakened depending on the temporal order of synaptic firing (Song et al., 2000; Taylor and Martin, 2009; Nolan et al., 2010). Theoretically, by repeating a motor task such as stepping STDP reinforces or weakens selected sensorimotor synapses and results in a neural network functioning with less variability. When a spinal animal is step trained, it seems likely that selected pathways are reinforced by the repetitive sensory input derived from stepping and the variance is reduced to, but not beyond, a critical level for effective 
motor control. A fixed-trajectory paradigm would seem to negatively influence learning attributable to the continuous interruptions by the robot and, in effect, by imposing more random (relative to the intrinsically defined sensorimotor events) responses and activation patterns of motor pools that are less effective in generating locomotion. Based on the STDP, there would be less selective reinforcement or weakening of synapses within the locomotor circuitry and, thus, a less effective stepping pattern with a fixed-trajectory versus an AAN paradigm.

Given that the amount of variance intrinsic to the spinal circuitry at any given stage of recovery after a spinal cord injury will differ between individuals, the ability to robotically aid modifications to the strategies of position control should be able to accommodate the optimal variance intrinsic to the circuitry at any particular point in time (Fig. 6). The present results show that limiting the robotic assistance during stepping to when the ankle moves outside a predetermined range decreases the chance of disrupting the muscle activation strategy "planned" by the spinal circuitry. The objective, therefore, should be to progressively reduce the variability to some critical level. A training paradigm that calculates the forced robotic adjustments in real time and changes the size and shape of the window to minimize disruptions of the locomotor circuitry, i.e., increasing the time that the robot is passive, theoretically should improve the efficiency of relearning how to step after a spinal cord injury.

Multiple factors determine the responsiveness of an individual with a spinal cord injury to a therapeutic intervention designed to improve locomotor function. If an individual has a motor incomplete injury and is receiving robotically mediated training, an important variable is the instructions given to the subject regarding "effort." Does the individual remain relaxed, exert forces on the robotic device, or attempt to minimize the forces imposed on the device? After a motor complete spinal cord injury, of course, these types of instructions are less important, but other variables become more relevant. For example, a critical factor will be the responsiveness of the spinal neuromotor circuitry to the robotic control. If the neural circuitry is not sufficiently active and responsive, the result will be neurologically passive, and it is likely that no significant improvement will occur. An additional possibility, however, is that, with a fixed trajectory, the neuromotor circuitry habituates and quickly becomes nonresponsive to the mechanical manipulation. Based on the present results, it would appear that the most appropriate instructions for the individual with an incomplete injury would be to attempt to follow the trajectory, which has a critical level of intrinsic variability, while minimizing the forces imposed on the robotic device. In an individual with a complete injury, a key element is to have a spinal circuitry that is responsive to the mechanical events imposed on the lower limbs. For example, if a subject is receiving antispastic medication, the responsiveness to the robotic manipulation will be minimized. Thus, although there should be some caution to the extent that the present results in complete spinal rats are clinically relevant, the principles derived from the present observations would appear to remain relevant, i.e., that some critical level of variability in the neural control is desirable in relearning effective stepping after a spinal cord injury. The question that remains is how much variability for a given individual with a given functional state is desirable.

\section{References}

Bialek W, Rieke F, de Ruyter van Steveninck RR, Warland D (1991) Reading a neural code. Science 252:1854-1857.
Cai LL, Fong AJ, Otoshi CK, Liang Y, Burdick JW, Roy RR, Edgerton VR (2006) Implications of assist-as-needed robotic step training after a complete spinal cord injury on intrinsic strategies of motor learning. J Neurosci 26:10564-10568.

Christou EA, Poston B, Enoka JA, Enoka RM (2007) Different neural adjustments improve endpoint accuracy with practice in young and old adults. J Neurophysiol 97:3340-3350.

Courtine G, Gerasimenko Y, van den Brand R, Yew A, Musienko P, Zhong H, Song B, Ao Y, Ichiyama RM, Lavrov I, Roy RR, Sofroniew MV, Edgerton VR (2009) Transformation of nonfunctional spinal circuits into functional states after the loss of brain input. Nat Neurosci 12:1333-1342.

De Leon RD, Acosta CN (2006) Effect of robotic-assisted treadmill training and chronic quipazine treatment on hindlimb stepping in spinally transected rats. J Neurotrauma 23:1147-1163.

De Leon RD, Hodgson JA, Roy RR, Edgerton VR (1998) Full weight-bearing hindlimb standing following stand training in the adult spinal cat. J Neurophysiol 80:83-91.

Dietz V, Müller R (2004) Degradation of neuronal function following a spinal cord injury: mechanisms and countermeasures. Brain 127:2221-2231.

Dominici N, Ivanenko YP, Lacquaniti F (2007) Control of foot trajectory in walking toddlers: adaptation to load changes. J Neurophysiol 97:27902801.

Edgerton VR, de Guzman CP, Gregor RJ, Roy RR, Hodgson JA, Lovely RG (1991) Trainability of the spinal cord to generate hindlimb stepping patterns in adult spinalized cats. In: Neurobiological basis of human locomotion (Shimamura TM, Grillner S, Edgerton VR, eds), pp 411-423. Tokyo: Japan Scientific Societies.

Edgerton VR, Kim SJ, Ichiyama RM, Gerasimenko YP, Roy RR (2006) Rehabilitative therapies after spinal cord injury. J Neurotrauma 23:560-570

Edgerton VR, Courtine G, Gerasimenko YP, Lavrov I, Ichiyama RM, Fong AJ, Cai LL, Otoshi CK, Tillakaratne NJ, Burdick JW, Roy RR (2008) Training locomotor networks. Brain Res Rev 57:241-254.

Emken JL, Harkema SJ, Beres-Jones JA, Ferreira CK, Reinkensmeyer DJ (2008) Feasibility of manual teach-and-replay and continuous impedance shaping for robotic locomotor training following spinal cord injury. IEEE Trans Biomed Eng 55:322-334.

Faisal AA, Selen LP, Wolpert DM (2008) Noise in the nervous system. Nat Rev Neurosci 9:292-303.

Fong AJ, Cai LL, Otoshi CK, Reinkensmeyer DJ, Burdick JW, Roy RR, Edgerton VR (2005) Spinal cord-transected mice learn to step in response to quipazine in treatment and robotic training. J Neurosci 25:11738-11747.

Frigon A, Rossignol S (2006) Functional plasticity following spinal cord lesions. Prog Brain Res 157:231-260.

Giszter SF, Davies MR, Graziani V (2008) Coordination strategies for limb forces during weight-bearing locomotion in normal rats, and in rats spinalized as neonates. Exp Brain Res 190:53-69.

Hausdorff JM (2005) Gait variability: methods, modeling and meaning. J Neuroeng Rehabil 2:19.

Hidler JM, Wall AE (2005) Alterations in muscle activation patterns during robotic-assisted walking. Clin Biomech (Bristol, Avon) 20:184-193.

Ichiyama RM, Courtine G, Gerasimenko YP, Yang GJ, van den Brand R, Lavrov IA, Zhong H, Roy RR, Edgerton VR (2008) Step training reinforces specific spinal locomotor circuitry in adult spinal rats. J Neurosci 28:7370-7375.

Israel JF, Campbell DD, Kahn JH, Hornby TG (2006) Metabolic costs and muscle activity patterns during robotic- and therapist-assisted treadmill walking in individuals with incomplete spinal cord injury. Phys Ther 86:1466-1478

Jezernik S, Schärer R, Colombo G, Morari M (2003) Adaptive robotic rehabilitation of locomotion: a clinical study in spinally injured individuals. Spinal Cord 41:657-666.

Kao MH, Wright BD, Doupe AJ (2008) Neurons in a forebrain nucleus required for vocal plasticity rapidly switch between precise firing and variable bursting depending on social context. J Neurosci 28:13232-13247.

Kiehn O (2006) Locomotor circuits in the mammalian spinal cord. Annu Rev Neurosci 29:279-306.

Kozlov A, Huss M, Lansner A, Kotaleski JH, Grillner S (2009) Simple cellular and network control principles govern complex patterns of motor behavior. Proc Natl Acad Sci U S A 106:20027-20032. 
Kubasak MD, Jindrich DL, Zhong H, Takeoka A, McFarland KC, MuñozQuiles C, Roy RR, Edgerton VR, Ramón-Cueto A, Phelps PE (2008) OEG implantation and step training enhance hindlimb-stepping ability in adult spinal transected rats. Brain 131:264-276.

Mandelblat-CerfY, Paz R, Vaadia E (2009) Trial-to-trial variability of single cells in motor cortices is dynamically modified during visuomotor adaptation. J Neurosci 29:15053-15062.

McDonnell MD, Abbott D (2009) What is stochastic resonance? Definitions, misconceptions, debates, and its relevance to biology. PLoS Comput Biol 5:e1000348.

Nolan CR, Wyeth G, Milford M, Wiles J (2010) The race to learn: spike timing and STDP can coordinate learning and recall in CA3. Hippocampus. Advance online publication. Retrieved May 25, 2010 doi:0.1002/hipo.20777.

Rossignol S (2000) Locomotion and its recovery after spinal injury. Curr Opin Neurobiol 10:708-716.

Shadlen MN, Newsome WT (1998) The variable discharge of cortical neu- rons: implications for connectivity, computation, and information coding. J Neurosci 18:3870-3896.

Song S, Miller KD, Abbott LF (2000) Competitive Hebbian learning through spiketiming-dependent synaptic plasticity. Nat Neurosci 3:919-926.

Stein RB, Gossen ER, Jones KE (2005) Neuronal variability: noise or part of the signal? Nat Rev Neurosci 6:389-397.

Taylor JL, Martin PG (2009) Voluntary motor output is altered by spiketiming-dependent changes in the human corticospinal pathway. J Neurosci 29:11708-11716.

Timoszyk WK, Nessler JA, Acosta C, Roy RR, Edgerton VR, Reinkensmeyer DJ, de Leon R (2005) Hindlimb loading determines stepping quantity and quality following spinal transection. Brain Res 1050:180-189.

Wirz M, Zemon DH, Rupp R, Scheel A, Colombo G, Dietz V, Hornby TG (2005) Effectiveness of automated locomotor training in patients with chronic incomplete spinal cord injury: a multicenter trial. Arch Phys Med Rehabil 86:672-680. 